BNL-HET-02/27

FSU-HEP-2002/1115

UB-HET-02/09

hep-ph/0211438

\title{
Associated Top Quark-Higgs Boson Production at the LHC
}

\author{
S. Dawson \\ Department of Physics, Brookhaven National Laboratory, \\ Upton, NY 11973-5000, USA \\ L. H. Orr \\ Department of Physics \& Astronomy, University of Rochester, \\ Rochester, NY 14627-0171, USA \\ L. Reina \\ Department of Physics, Florida State University, \\ Tallahassee, FL 32306-4350, USA \\ D. Wackeroth \\ Department of Physics, State University of New York at Buffalo, \\ Buffalo, NY 14260-1500, USA
}

\begin{abstract}
We compute the $\mathcal{O}\left(\alpha_{s}^{3}\right)$ inclusive cross section for the process $p p \rightarrow t \bar{t} h$ in the Standard Model, at $\sqrt{s_{H}}=14 \mathrm{TeV}$. The next-to-leading order corrections drastically reduce the renormalization and factorization scale dependence of the Born cross section and increase the total cross section for renormalization and factorization scales larger than $m_{t}$. These corrections have important implications for models of new physics involving the top quark.
\end{abstract}




\title{
Associated Top Quark-Higgs Boson Production at the LHC
}

\author{
S. Dawson, ${ }^{1}$ L. H. Orr, ${ }^{2}$ L. Reina,${ }^{3}$ and D. Wackeroth ${ }^{4}$ \\ ${ }^{1}$ Department of Physics, Brookhaven National Laboratory, Upton, NY 11973-5000, USA \\ ${ }^{2}$ Department of Physics \& Astronomy, University of Rochester, Rochester, NY 14627-0171, USA \\ ${ }^{3}$ Department of Physics, Florida State University, Tallahassee, FL 32306-4350, USA \\ ${ }^{4}$ Department of Physics, State University of New York at Buffalo, Buffalo, NY 14260-1500, USA
}

(Dated: November 2, 2018)

\begin{abstract}
We compute the $\mathcal{O}\left(\alpha_{s}^{3}\right)$ inclusive cross section for the process $p p \rightarrow t \bar{t} h$ in the Standard Model, at $\sqrt{s_{H}}=14 \mathrm{TeV}$. The next-to-leading order corrections drastically reduce the renormalization and factorization scale dependence of the Born cross section and increase the total cross section for renormalization and factorization scales larger than $m_{t}$. These corrections have important implications for models of new physics involving the top quark.
\end{abstract}

PACS numbers: $14.80 . \mathrm{Bn}, 12.38 . \mathrm{Bx}, 12.15 .-\mathrm{y}, 13.85 .-\mathrm{t}$

1. One of the major goals of the LHC is uncovering the mechanisms of electroweak symmetry breaking and the generation of fermion masses. In the Standard Model of particle physics, the masses of gauge bosons and fermions are generated by a single scalar field. After spontaneous symmetry breaking, a neutral CP-even Higgs boson, $h$, remains as a physical particle. The fermion masses then arise through couplings to the Higgs boson. In the Standard Model, this coupling is directly proportional to the fermion mass. Since the top quark is the most massive quark, its coupling to the Higgs boson is particularly sensitive to the underlying physics.

The associated production of a Higgs boson with a $t \bar{t}$ pair at the LHC, $p p \rightarrow t \bar{t} h$, will play a very important role in the $115 \mathrm{GeV} \leq M_{h} \leq 140 \mathrm{GeV}$ Higgs boson mass range, both for discovery and for precision measurements of the Higgs boson couplings. This process will provide a direct measurement of the top-quark Yukawa coupling and will be instrumental in determining ratios of Higgs boson couplings in a model independent way [1, 2. Such measurements could help to distinguish a SM Higgs boson from more complex Higgs sectors, e.g., as predicted by supersymmetry, and shed light on the details of the generation of fermion masses.

In order to interpret the evidence for $t \bar{t} h$ production and the measurement of the $t \bar{t} h$ coupling as a verification of the Standard Model or as a signal for new physics, it is necessary to have a precise prediction for the cross section. QCD corrections are expected to be important and are crucial in order to reduce the dependence of the cross section on the arbitrary renormalization and factorization scales. In this letter, we present the next-toleading-order (NLO) QCD corrections to the total cross section for $p p \rightarrow t \bar{t} h$ at the LHC. Our results are in very good agreement with those of Ref. [3] within the statistical errors. Results for the Fermilab Tevatron have been presented elsewhere [3, 因, 5].
2. The inclusive total cross section for $p p \rightarrow t \bar{t} h$ at $\mathcal{O}\left(\alpha_{s}^{3}\right)$ can be written as:

$$
\begin{aligned}
& \sigma_{N L O}(p p \rightarrow t \bar{t} h)=\sum_{i j} \frac{1}{1+\delta_{i j}} \int d x_{1} d x_{2} \\
& \cdot\left[\mathcal{F}_{i}^{p}\left(x_{1}, \mu\right) \mathcal{F}_{j}^{p}\left(x_{2}, \mu\right) \hat{\sigma}_{N L O}^{i j}\left(x_{1}, x_{2}, \mu\right)+(1 \leftrightarrow 2)\right],
\end{aligned}
$$

where $\mathcal{F}_{i}^{p}$ is the NLO parton distribution function for parton $i$ in a proton, defined at a factorization scale $\mu_{f}=\mu$, and $\hat{\sigma}_{N L O}^{i j}$ is the $\mathcal{O}\left(\alpha_{s}^{3}\right)$ parton level total cross section for incoming partons $i$ and $j$, made of the channels $q \bar{q}, g g \rightarrow t \bar{t} h$, and $(q, \bar{q}) g \rightarrow t \bar{t} h(q, \bar{q})$, and renormalized at the scale $\mu_{r}$ which we also take to be $\mu_{r}=\mu$. At the LHC, the dominant contribution is from the gluon-gluon initial state, although the other contributions cannot be neglected and are included in this calculation.

The NLO parton-level total cross section, $\hat{\sigma}_{N L O}^{i j}$, consists of the $\mathcal{O}\left(\alpha_{s}^{2}\right)$ Born cross section, $\hat{\sigma}_{L O}^{i j}$, and the $\mathcal{O}\left(\alpha_{s}\right)$ corrections to the Born cross section, $\delta \hat{\sigma}_{N L O}^{i j}$, including the effects of mass factorization. $\delta \hat{\sigma}_{N L O}^{i j}$ contains virtual and real corrections to the parton-level $t \bar{t} h$ production processes, $q \bar{q} \rightarrow t \bar{t} h$ and $g g \rightarrow t \bar{t} h$, and the tree-level $(q, \bar{q}) g$ initiated processes, $(q, \bar{q}) g \rightarrow t \bar{t} h(q, \bar{q})$, which are of the same order in $\alpha_{s}$. It can be written as the sum of two terms:

$$
\begin{aligned}
& \delta \hat{\sigma}_{N L O}^{i j}=\hat{\sigma}_{v i r t}^{i j}+\hat{\sigma}_{\text {real }}^{i j}= \\
& \int d\left(P S_{3}\right) M(i j \rightarrow t \bar{t} h)+\int d\left(P S_{4}\right) M(i j \rightarrow t \bar{t} h+k),
\end{aligned}
$$

where $M(i j \rightarrow t \bar{t} h)$ and $M(i j \rightarrow t \bar{t} h+k)($ for $k=g, q, \bar{q})$ are respectively the matrix elements squared for the $\mathcal{O}\left(\alpha_{s}^{3}\right) 2 \rightarrow 3$ and $2 \rightarrow 4$ scattering processes averaged over the initial degrees of freedom and summed over the final ones, while $d\left(P S_{3}\right)$ and $d\left(P S_{4}\right)$ denote the integration over the corresponding three/four particle phase space. The $(q, \bar{q}) g$ initial state contributes only to $\hat{\sigma}_{\text {real }}^{i j}$.

The main challenges in the calculation come from the presence in the virtual corrections of pentagon diagrams with several massive external and internal particles, and 
from the computation of the real part in the presence of infrared singularities.

3. The $\mathcal{O}\left(\alpha_{s}\right)$ virtual corrections to the tree level $i j \rightarrow t \bar{t} h(i j=q \bar{q}, g g)$ processes consist of self-energy, vertex, box, and pentagon diagrams. The calculation of the virtual corrections to the $q \bar{q}$ initial state is described in Ref. [5]. The basic method is to reduce each diagram to a sum of scalar integrals of the form,

$$
\int \frac{d^{d} k}{(2 \pi)^{d}} \prod_{i=0}^{n \leq 4} \frac{1}{\left[\left(k+p_{i}\right)^{2}-m_{i}^{2}\right]} ; p_{0}=0,
$$

that may contain both ultraviolet (UV) and infrared (IR) singularities. The finite scalar integrals are evaluated by using the method described in Ref. [6] and cross checked with the numerical package FF [7]. The scalar integrals that exhibit UV and/or IR divergences are calculated analytically. Both the UV and IR divergences are extracted by using dimensional regularization in $d=4-2 \epsilon$ dimensions. The UV divergences are then removed by introducing a suitable set of counterterms, as described in detail in Ref. [5]. The IR divergences are cancelled by the analogous singularities in the soft and collinear part of the real gluon emission cross section.

The most difficult integrals arise from the IR divergent pentagon diagrams with several massive particles. In Refs. 4, 5, 8] we calculated the pentagon scalar integrals as linear combinations of scalar box integrals using the method of Ref. 9, 10]. For the $g g$ initiated process we also used the method of Ref. 6] and found perfect agreement between the results of the two methods. The virtual corrections to the $g g$ initiated process have an additional complication with respect to the $q \bar{q}$ case because of the presence of pentagon tensor integrals with rank higher than one. Pentagon tensor integrals can give rise to numerical instabilities due to the dependence on inverse powers of the Gram determinant (GD):

$$
\begin{aligned}
\mathrm{GD}\left(p_{1}+p_{2} \rightarrow \sum_{i=3}^{5} p_{i}\right)=-\frac{\left[s-\left(2 m_{t}+M_{h}\right)^{2}\right]}{64} \times \\
\\
\quad\left[M_{h}^{4}+\left(s-s_{45}\right)^{2}-2 M_{h}^{2}\left(s+s_{45}\right)\right] s s_{45} \times \\
\quad \sin ^{2} \theta_{45} \sin ^{2} \phi_{45} \sin ^{2} \theta,
\end{aligned}
$$

where the 3-particle phase space has been expressed in terms of a time-like invariant $s_{45}=\left(p_{4}+p_{5}\right)^{2}$, polar angles, $\theta_{45}, \theta$ and azimuthal angles, $\phi_{45}, \phi$, and $s=x_{1} x_{2} s_{H}$ is the partonic center-of-mass energy squared. As can be seen in Eq. (4), the Gram determinant vanishes when two momenta become degenerate, i.e. at the boundaries of phase space. These are spurious divergences, which cause serious numerical difficulties. We use two methods to overcome this problem and find mutual agreement within the statistical uncertainty of the phase space integration:

- Impose kinematic cuts to avoid the phase space regions where the Gram determinant vanishes. Then apply an extrapolation procedure from the numerically safe to the numerically unsafe region.

- Eliminate all pentagon tensor integrals by cancelling terms in the numerator against the propagators wherever possible, after interfering the pentagon amplitude with the Born matrix element. The resulting expressions are very large, but numerically stable.

4. The $\mathcal{O}\left(\alpha_{s}\right)$ corrections to the Born cross sections for the $q \bar{q}$ and $g g$ initial states due to real gluon emission, as well as the $(q, \bar{q}) g$ initiated processes, have been computed using both a two cutoff [11] and a single cutoff [12, 13, 14] implementation of the phase space slicing (PSS) algorithm. In both PSS methods, the real contribution to the NLO rate is computed analytically below the cutoff(s) and numerically above the cutoff(s) and the final result is independent of these arbitrary parameters. When studying the cutoff dependence of $\sigma_{N L O}$, it is crucial to choose the cutoff(s) small enough to justify the analytical calculations of the IR divergent contributions to $\hat{\sigma}_{\text {real }}^{i j}$, but not so small as to cause numerical instabilities. Finding agreement between the two PSS approaches is therefore a strong check of the accuracy of the calculation.

In the two cutoff PSS algorithm, the contributions of $q \bar{q}, g g \rightarrow t \bar{t} h+g$ to $\hat{\sigma}_{\text {real }}^{i j}$ are first divided into a soft and a hard part,

$$
\hat{\sigma}_{\text {real }}^{i j}=\hat{\sigma}_{\text {soft }}^{i j}+\hat{\sigma}_{\text {hard }}^{i j},
$$

where soft and hard refer to the energy of the final state radiated gluon. This division into hard and soft contributions depends on a soft cutoff, $\delta_{s}$, such that the energy of the radiated gluon in the partonic center-of-mass frame is considered soft if $E_{g} \leq \delta_{s} \frac{\sqrt{s}}{2}$. The eikonal approximation to the soft matrix elements can be taken and the integral over the soft degrees of freedom performed analytically. Since the $(q, \bar{q}) g$ initiated process does not develop soft singularities, it only contributes to $\hat{\sigma}_{\text {hard }}^{q g}$ and no soft cutoff is applied.

The hard contribution to $i j \rightarrow t \bar{t} h+k(k=g, q, \bar{q})$ is further divided into a hard/collinear part, $\hat{\sigma}_{\text {hard/coll }}^{i j}$, and a hard/non collinear part, $\hat{\sigma}_{\text {hard/non-coll }}^{i j}$. For $q \bar{q}$ and $g g$ initiated processes the hard/collinear region is defined as the region where the energy of the final state gluon is $E_{g}>\delta_{s} \frac{\sqrt{s}}{2}$ and the gluon is radiated from the initial massless parton at an angle $\theta_{i g}$, in the $i j$ center-ofmass frame, such that $\left(1-\cos \theta_{g i}\right) \leq \delta_{c}$, for an arbitrary small collinear cutoff $\delta_{c}$. The matrix element squared in the hard/collinear limit is calculated using the leading pole approximation, resulting into the convolution of the unregulated Altarelli-Parisi splitting functions $P_{q \bar{q}}$ or $P_{g g}$ with the corresponding tree level matrix elements squared. In the same way, the matrix elements squared for the $(q, \bar{q}) g$ initiated processes are described, in the 
collinear region $\left(1-\cos \theta_{(q, \bar{q}) i}\right) \leq \delta_{c}$, as convolutions of the unregulated Altarelli-Parisi splitting functions $P_{g q}$ and $P_{q g}$ with the $q \bar{q}$ and $g g$ tree-level matrix elements, respectively. The integration over the angular degrees of freedom can then be performed analytically.

The hard gluon emission from the final massive quarks never belongs to the hard/collinear region. The contribution from the hard/non collinear region is finite and is computed numerically, using standard Monte Carlo integration techniques.

Both $\hat{\sigma}_{\text {soft }}^{i j}$ and $\hat{\sigma}_{\text {hard }}^{i j}$ depend on the arbitrary cutoff $\delta_{s}$, and $\hat{\sigma}_{\text {hard/coll }}^{i j}$ and $\hat{\sigma}_{\text {hard/non-coll }}^{i j}$ also depend on $\delta_{c}$. However, the real hadronic cross section, $\sigma_{\text {real }}$, after mass factorization, is cutoff independent. The cutoff independence of the NLO cross section, $\sigma_{N L O}$, is shown in Fig. 1, where we assume $\delta_{c}=\delta_{s} / 100$ and we let $\delta_{s}$ vary between $10^{-5}$ and $10^{-3}$. The hadronic cross sections $\sigma_{\text {soft }}+\sigma_{\text {hard/coll }}$ and $\sigma_{\text {hard/non-coll }}$ include the contributions of all three $t \bar{t} h$ production channels, $q \bar{q}, g g \rightarrow t \bar{t} h g$ and $(q, \bar{q}) g \rightarrow t \bar{t} h(q, \bar{q})$. For $\delta_{s}$ in the range $10^{-5}-10^{-3}$ and for $\delta_{c}=\delta_{s} / 100$, a clear plateau is reached and the result is independent of $\delta_{s}$ and $\delta_{c}$.

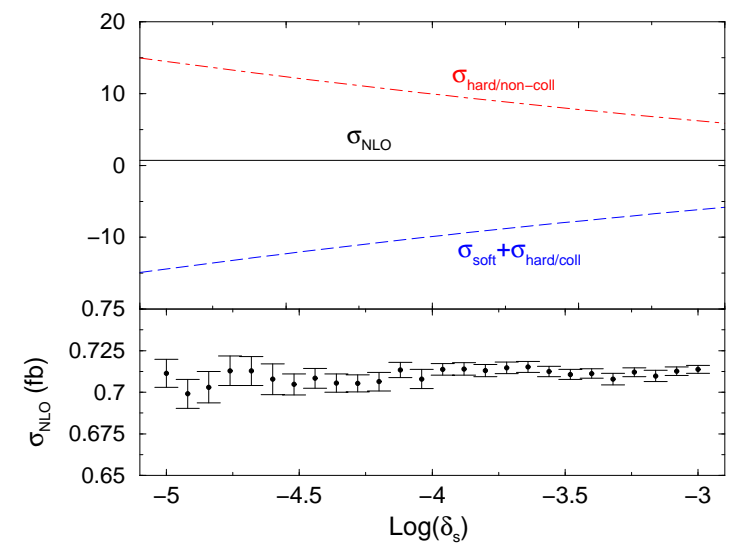

FIG. 1: $\sigma_{N L O}(p p \rightarrow t \bar{t} h)$ calculated in the two cutoff PSS approach when varying the soft cutoff $\delta_{s}$ in the range $10^{-5}-10^{-3}$, with the collinear cutoff set to $\delta_{c}=\delta_{s} / 100$, at $\sqrt{s_{H}}=14 \mathrm{TeV}$, for $M_{h}=120 \mathrm{GeV}$ and $\mu=m_{t}$. The upper plot shows the cancellation of the $\delta_{s}, \delta_{c}$ dependence between $\sigma_{\text {soft }}+\sigma_{\text {hard } / \text { coll }}$ and $\sigma_{\text {hard/non-coll }}$. The lower plot shows the dependence of $\sigma_{N L O}$ on $\delta_{s}, \delta_{c}$, with the corresponding statistical errors.

An alternative method of isolating both soft and collinear singularities is to divide the phase space of the final state partons into two regions, according to whether all partons can be resolved or not.

The single cutoff PSS technique defines the IR divergent region as that where the final state parton $k$ emitted from parton $i$ is not resolved and

$$
s_{i k}=2 p_{i} \cdot p_{k}<s_{\min }
$$

for an arbitrarily small value of the cutoff $s_{\min }$. The partonic real cross section is then written as

$$
\hat{\sigma}_{\text {real }}^{i j}=\hat{\sigma}_{i r}^{i j}+\hat{\sigma}_{\text {hard }}^{i j}=\hat{\sigma}_{i r}^{c, i j}+\hat{\sigma}_{\text {crossing }}^{i j}+\hat{\sigma}_{\text {hard }}^{i j},
$$

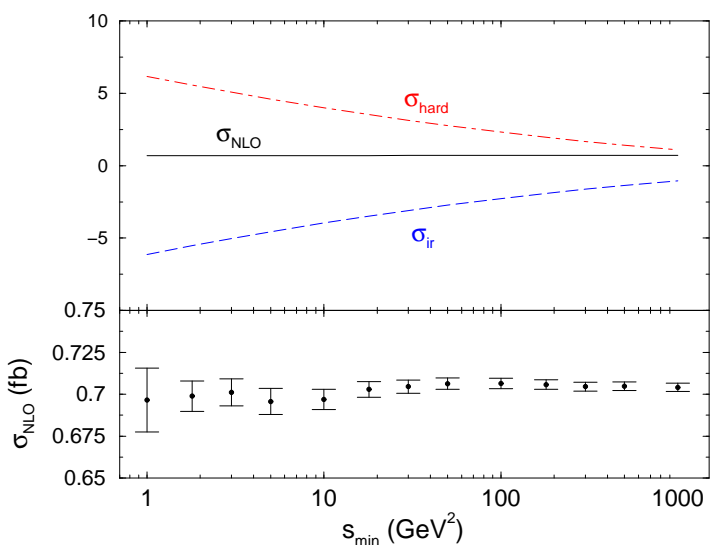

FIG. 2: Dependence of $\sigma_{N L O}(p p \rightarrow t \bar{t} h)$ on the arbitrary cutoff $s_{\min }$ of the one cutoff PSS method at $\sqrt{s_{H}}=14 \mathrm{TeV}$, for $M_{h}=120 \mathrm{GeV}$ and $\mu=m_{t}$. The upper plot shows the cancellation of the $s_{\text {min }}$ dependence between $\sigma_{i r}=\sigma_{i r}^{c}+\sigma_{\text {crossing }}$, and $\sigma_{\text {hard }}$. The lower plot shows the dependence of $\sigma_{N L O}$ on $s_{\text {min }}$, with the corresponding statistical errors.

where $\hat{\sigma}_{i r}^{i j}$ includes both soft and collinear singularities and $\hat{\sigma}_{\text {hard }}^{i j}$ is finite. The IR divergent contribution $\left(\hat{\sigma}_{i r}^{c, i j}\right)$ is computed from the crossed process, $h \rightarrow i j t \bar{t}+k$, where $i j$ denote the initial state partons and $k=g, q, \bar{q}$. This permits a straightforward decomposition of the amplitude in terms of color ordered amplitudes. Using the factorization properties of both the color ordered amplitudes and the gluon/quark phase space in the soft/collinear limits, the IR divergent contribution can be extracted analytically. The factorization of soft and collinear singularities for color ordered amplitudes has been discussed in the literature mainly for the leading color terms, $\mathcal{O}\left(N_{c}\right)$ 12, 13, 14. In our case, however, the inclusion of the sub-leading terms in $1 / N_{c}$ is crucial. For the $q \bar{q}$ initial state, these terms were first calculated in Ref. [5].

An additional contribution to $\hat{\sigma}_{i r}^{i j}\left(\hat{\sigma}_{\text {crossing }}^{i j}\right)$ arises when crossing partons $i$ and $j$ back to the initial state, due to the mismatch between the collinear gluon radiation from initial and final state partons. $\hat{\sigma}_{\text {crossing }}^{i j}$ contains collinear divergences which are cancelled by the parton distribution counterterms when the parton cross section is convoluted with the PDF's. In Fig. 2, the independence of $\sigma_{N L O}$ from the cutoff $s_{\min }$ is demonstrated. Again, the hadronic cross sections $\sigma_{i r}^{c}+\sigma_{\text {crossing }}$ and $\sigma_{\text {hard }}$ include the contributions from all initial states, $q \bar{q}, g g$ and $(q, \bar{q}) g$. Together with Ref. [5], this is the first application of the single cutoff PSS approach the calculation of a cross section involving more than one massive particle in the final state.

The numerical results of both PSS methods agree within the statistical errors and within the systematic errors of the applied soft and collinear approximations. In Ref. [3], the dipole subtraction formalism has been used to extract the IR singularities of the real part. The agreement between these three very different treatments 
of the real IR singularities represents a powerful check of the corresponding NLO calculations.

5. Our numerical results are found using CTEQ4M parton distribution functions for the calculation of the NLO cross section, and CTEQ4L parton distribution functions for the calculation of the lowest order cross section [15. The NLO (LO) cross section is evaluated using the $2(1)$-loop evolution of $\alpha_{s}(\mu)$. The top quark mass is taken to be $m_{t}=174 \mathrm{GeV}$ and $\alpha_{s}^{N L O}\left(M_{Z}\right)=0.116$.

In Fig. 3 we show, for $M_{h}=120 \mathrm{GeV}$, the dependence of $\sigma_{L O}$ and $\sigma_{N L O}$ on the arbitrary renormalization/factorization scale $\mu=\mu_{r}=\mu_{f}$. For scales $\mu$ larger than $m_{t}$ the NLO result is significantly larger than the lowest order result.

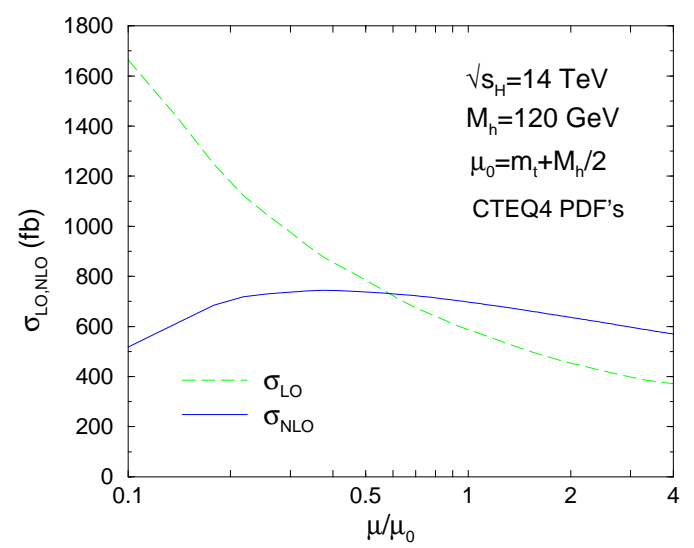

FIG. 3: $\sigma_{L O, N L O}(p p \rightarrow t \bar{t} h)$ as functions of the renormalization/factorization scale $\mu$, at $\sqrt{s_{H}}=14 \mathrm{TeV}$, for $M_{h}=$ $120 \mathrm{GeV}$.

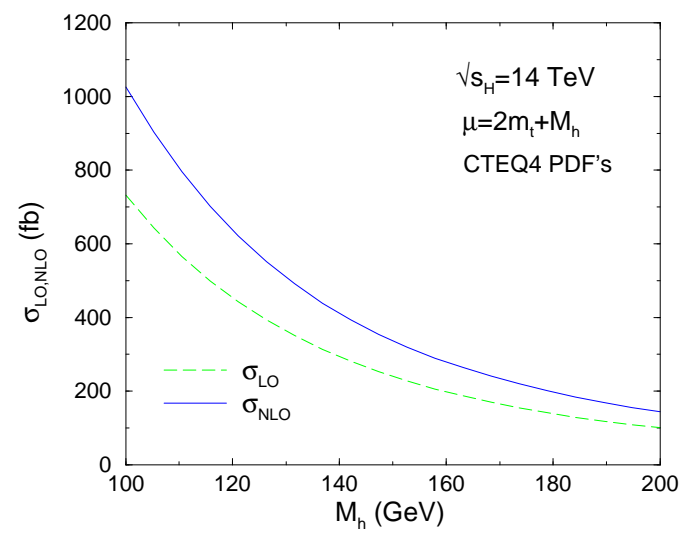

FIG. 4: $\sigma_{N L O}$ and $\sigma_{L O}$ for $p p \rightarrow t \bar{t} h$ as functions of $M_{h}$, at $\sqrt{s_{H}}=14 \mathrm{TeV}$, for $\mu=2 m_{t}+M_{h}$.

Fig. 1 shows both the LO and the NLO total cross section for $p p \rightarrow t \bar{t} h$ at $\sqrt{s_{H}}=14 \mathrm{TeV}$, as functions of $M_{h}$, for a representative value of the renormalization/factorization scale, $\mu=2 m_{t}+M_{h}$. We estimate the remaining theoretical error, due to the residual $\mu$ dependence, to the parton distribution functions, and to the experimental error on $m_{t}$, to be of the order of 15$20 \%$. In comparison, the statistical error on the numerical integration is negligible, due to the high statistics used in evaluating the total cross section.

6. The NLO QCD corrections to the Standard Model process $p p \rightarrow t \bar{t} h$, at $\sqrt{s_{H}}=14 \mathrm{TeV}$ increase the LO cross section by a factor of $1.2-1.4$ for renormalization/factorization scales in the range $m_{t}+M_{h} / 2 \leq \mu \leq$ $4 m_{t}+2 M_{h}$ and Higgs boson masses in the range considered in this paper. The NLO result shows a drastically reduced scale dependence as compared to the Born result and leads to increased confidence in predictions based on these results. The techniques developed in this calculation can now be applied to the study of the associated $b \bar{b} h$ production at both the LHC and the Tevatron.

We thank U. Baur, Z. Bern, and F. Paige for valuable discussions and encouragement. We are grateful to the Authors of Ref. [3] for a detailed comparison of the results. The work of S.D. (L.H.O., L.R.) is supported in part by the U.S. Department of Energy under grant DE-AC02-76CH00016 (DE-FG-02-91ER40685, DE-FG02-97ER41022).

[1] A. Belyaev and L. Reina, JHEP 08, 041 (2002), hep$\mathrm{ph} / 0205270$.

[2] D. Zeppenfeld (2002), hep-ph/0203123.

[3] W. Beenakker, S. Dittmaier, M. Krämer, B. Plümper, M. Spira, and P. Zerwas, Phys. Rev. Lett. 87, 201805 (2001), hep-ph/0107081; hep-ph/0211352.

[4] L. Reina and S. Dawson, Phys. Rev. Lett. 87, 201804 (2001), hep-ph/0107101.

[5] L. Reina, S. Dawson, and D. Wackeroth, Phys. Rev. D65, 053017 (2002), hep-ph/0109066.

[6] A. Denner, Fortschr. Phys. 41, 307 (1993).

[7] G. J. van Oldenborgh and J. A. M. Vermaseren, Z. Phys. C46, 425 (1990).

[8] S. Dawson, L.H. Orr, L. Reina, and D. Wackeroth (2002), hep-ph/0210109.

[9] Z. Bern, L. J. Dixon, and D. A. Kosower, Phys. Lett. B302, 299 (1993), erratum-ibid. B318, 649 (1993), hep$\mathrm{ph} / 9212308$.

[10] Z. Bern, L. J. Dixon, and D. A. Kosower, Nucl. Phys. B412, 751 (1994), hep-ph/9306240.

[11] B. W. Harris and J. F. Owens, Phys. Rev. D65, 094032 (2002), hep-ph/0102128.

[12] W. T. Giele and E. W. N. Glover, Phys. Rev. D46, 1980 (1992).

[13] W. T. Giele, E. W. N. Glover, and D. A. Kosower, Nucl. Phys. B403, 633 (1993), hep-ph/9302225.

[14] S. Keller and E. Laenen, Phys. Rev. D59, 114004 (1999), hep-ph/9812415.

[15] H. L. Lai et al., Phys. Rev. D55, 1280 (1997), hep$\mathrm{ph} / 9606399$. 